\title{
A Dynamic Model Free Observer Based Output Feedback Tracking Control of Robot Manipulators in Task-Space
}

\author{
Necati Cobanoglu, Kamil Cetin, Enver Tatlicioglu ${ }^{\star}$, and Erkan Zergeroglu
}

\begin{abstract}
This paper addresses the output feedback endeffector position tracking control of robotic manipulators. Specifically, via the design of a novel dynamic model independent observer constructed through a Lyapunov type analysis and under the assumption that the manipulator Jacobian is always invertible, we propose a model based nonlinear controller which ensures asymptotic robot end-effector tracking without the need of joint and/or task space velocity measurements. Simulation results are included to illustrate the performance and effectiveness of the proposed method.
\end{abstract}

\section{INTRODUCTION}

In robotic applications, the dynamic model of the robot manipulator is commonly formulated in joint-space, while the desired motion of the robot manipulator is usually described in task-space (also referred as the Cartesian space) where the end-effector of the robot manipulator performs the actual task. One method to address this control problem is first to obtain the desired joint-space trajectory and then design a controller in the joint-space. This method is commonly called indirect task-space control [1]. The requirement of the solution of the inverse kinematics online is a shortcoming of this method. Alternatively, the tracking controller may be designed in the task-space by aiming to develop a feedback loop where the end-effector of the robot manipulator tracks the desired end-effector trajectory. This method is commonly called direct task-space control [1].

As can be seen in the recent review papers by [2] and [3], a good amount of past research was devoted to designing direct task-space controllers. There are many past works that addressed different task-space tracking control problems. In [4], dynamic control of robot manipulators in task-space was introduced. Hsu et al in [5] designed a feedback linearizing controller for task-space tracking control of redundant robot manipulators. Adaptive control of redundant robot manipulators while ensuring practical sub-task tracking was presented in [6]. Tatlicioglu et al in [7] designed an adaptive feedback linearizing controller for redundant robot manipulators while achieving secondary control objectives. Yazarel and Cheah in [8] designed an adaptive controller when some part of the dynamics and the kinematics were uncertain. Controllers were

N. Cobanoglu and E. Tatlicioglu are with the Department of Electrical \& Electronics Engineering, Izmir Institute of Technology, Izmir, 35430 Turkey (Phone: +90 (232) 7506536; Fax: +90 (232) 7506599; E-mail: [necaticobanoglu,kamilcetin,envertatlicioglu]@iyte.edu.tr).

E. Zergeroglu is with the Department of Computer Engineering, Gebze Technical University, 41400, Gebze, Kocaeli, Turkey (Email: e.zerger@gtu.edu.tr).

E. Tatlicioglu is partially funded by The Scientific and Technological Research Council of Turkey via grant number 115E726.

${ }^{\star}$ To whom all the correspondence should be addressed. designed for robot manipulators with uncertain Jacobian matrix [9], [10], [11]. Approximate Jacobian controllers were then designed in [12], [13]. [14] and [15] designed adaptive controllers when both kinematic and dynamic models included parametric uncertainties. Dogan et al in [16] designed a learning controller in task-space for applications that required periodic desired task. A high-gain robust controller was designed in [17] for redundant robot manipulators. Cetin et al in [18] designed a task-space tracking controller that did not require dynamic model parameters. Recently, variable structure type controllers were designed for taskspace tracking control of robot manipulators [19], [20], [21]. While aspects such as uncertain dynamics, uncertain Jacobian, etc were addressed heavily in the literature, most of these task-space tracking control methods were based on full state feedback that required both joint position and joint velocity measurements. This is a weakness of some of the works as most of the industrial robots are not equipped with joint velocity measurements devices and differentiating joint position measurements introduces high frequency signals to the control input. In order to eliminate the requirement of the joint velocity measurements for the task-space tracking control of the robot manipulator, there are some works that developed output feedback controllers by designing joint velocity observers [22], [23], [24]. In [22], [23], authors developed exact model knowledge controllers with the velocity observer to eliminate the joint velocity measurements. [24] designed a quaternion-based output feedback controller based on a joint velocity observer for task-space tracking control of the robot manipulators. The main shortcoming of [22], [23], [24] was that the joint velocity observation relied on accurate knowledge or robot dynamics. Utilizing observers that do not require dynamic model knowledge (such as high gain observers in [25]) usually loses asymptotic tracking.

In this paper, we propose a novel output feedback controller for the task-space, end-effector tracking problem of robotic manipulators. To eliminate the need of joint velocity measurements a dynamic model independent, joint velocity observer formulation is constructed via a Lyapunov type analysis. Due to the dynamical model independent nature of the observation formulation, the proposed method can be used as a stepping stone for further designs where accurate knowledge of the system dynamics is not available. Asymptotic stability of the controller/observer couple is guaranteed via Lyapunov type arguments and numerical studies utilizing the dynamic model of a two degree of freedom planar robot manipulator are performed to illustrate the performance and 
viability of the proposed method.

\section{Kinematic And Dynamic Models}

Kinematic formulation of an $n$ degree of freedom robot manipulator is given as,

$$
x=f(q)
$$

where $q(t) \in \mathbb{R}^{n}$ is joint position vector, $x(t) \in \mathbb{R}^{n}$ is the end-effector position of the robot manipulator and $f: \mathbb{R}^{n} \rightarrow$ $\mathbb{R}^{n}$ denotes forward kinematics. The differential kinematics, obtained by taking the time derivative of the kinematics is also obtained as

$$
\dot{x}=J \dot{q}
$$

where $\dot{q}(t) \in \mathbb{R}^{n}$ denotes the joint velocity vector and $J(q) \in$ $\mathbb{R}^{n \times n}$ is the Jacobian matrix defined as,

$$
J \triangleq \frac{\partial f}{\partial q} .
$$

Here we assumed that the Jacobian matrix defined in (3) is a square matrix. That is the sizes of the task-space and the joint space of the robotic manipulator are same. It is wellknown that, this is not necessarily the case for all robotic operations. However for the sake and ease of presentation we have selected Jacobian matrix as a square matrix, we would like to note that the formulation presented in the next section can also be used with pseudo-inverse formulations for the Jacobian matrix.

Assumption 1: $J(q)$ is full rank $\forall q(t)$, hence $J(q)$ is invertible and is away from kinematic singularities.

Assumption 2: There exists inverse kinematics function $h: \mathbb{R}^{n} \rightarrow \mathbb{R}^{n}$ such that ${ }^{1}$

$$
h(x)=q .
$$

In our controller development we will also make use of the following properties which are required to obtain the corresponding bounds for the auxiliary terms introduced later in our design steps;

Property 1: The kinematic terms satisfy the following properties [26]

$$
\begin{aligned}
\|h(a)-h(b)\| & \leq \zeta_{h 1}\|a-b\| \\
\|J(q)\|_{i \infty} & \leq \zeta_{J 1} \\
\left\|J^{-1}(q)\right\|_{i \infty} & \leq \zeta_{J 2} \\
\left\|J^{-1}(a)-J^{-1}(b)\right\|_{i \infty} & \leq \zeta_{J 3}\|a-b\| \quad \forall a, b \in \mathbb{R}^{n}(8)
\end{aligned}
$$

where $\zeta_{h 1}, \zeta_{J 1}, \zeta_{J 2}, \zeta_{J 3}$ are known positive bounding constants and $\|\cdot\|_{i \infty}$ denotes the induced infinity norm of matrix.

Property 2: The time derivative of the inverse Jacobian, defined as $W_{J}(q, \dot{q}) \triangleq \frac{d}{d t}\left(J^{-1}(q)\right) \in \mathbb{R}^{n \times n}$, satisfies the following properties [26]

$$
\begin{aligned}
W_{J}(q, a) b & =W_{J}(q, b) a \\
\left\|W_{J}(a, b)\right\|_{i \infty} & \leq \zeta_{J 4}\|b\| \\
\left\|W_{J}(a, b)-W_{J}(c, b)\right\|_{i \infty} & \leq \zeta_{J 5}\|a-c\|\|b\|
\end{aligned}
$$

\footnotetext{
${ }^{1}$ While the inverse kinematic at position level is introduced in (4), the subsequent controller will be designed not to require it.
}

$\forall a, b, c \in \mathbb{R}^{n}$ where $\zeta_{J 4}, \zeta_{J 5}$ are known positive bounding constants.

The dynamic model of an $n$ degree of freedom revolute joint robot manipulator is given as,

$$
M(q) \ddot{q}+V_{m}(q, \dot{q}) \dot{q}+G(q)+F_{d} \dot{q}=\tau
$$

where $\ddot{q}(t) \in \mathbb{R}^{n}$ is the joint acceleration vector, $M(q) \in$ $\mathbb{R}^{n \times n}$ is the inertia matrix, $V_{m}(q, \dot{q}) \in \mathbb{R}^{n \times n}$ represents the centripetal Coriolis effects, $G(q) \in \mathbb{R}^{n}$ represents the gravitational effects, $F_{d} \in \mathbb{R}^{n \times n}$ denotes the constant frictional effects, and $\tau(t) \in \mathbb{R}^{n}$ is the control input torque.

Property 3: The inertia matrix is positive definite and symmetric and satisfies the following inequalities [27]

$$
\begin{aligned}
\zeta_{M 1} I_{n} \leq M(q) & \leq \zeta_{M 2} I_{n} \\
\frac{1}{\zeta_{M 2}} I_{n} \leq M^{-1}(q) & \leq \frac{1}{\zeta_{M 1}} I_{n} \\
\|M(q)\|_{i \infty} & \leq \zeta_{M 2} \\
\left\|M^{-1}(q)\right\|_{i \infty} & \leq \frac{1}{\zeta_{M 1}}
\end{aligned}
$$

where $\zeta_{M 1}, \zeta_{M 2}$ are known positive bounding constants and $I_{n} \in \mathbb{R}^{n \times n}$ is the identity matrix.

Property 4: The time derivative of the inertia matrix and the centripetal Coriolis matrix satisfy the following property [27]

$$
a^{T}\left(\dot{M}-2 V_{m}\right) a=0 \quad \forall a \in \mathbb{R}^{n} .
$$

Property 5: The dynamic terms in (12) satisfy the following properties [27]

$$
\begin{aligned}
V_{m}(q, a) b & =V_{m}(q, b) a \\
\left\|V_{m}(q, a)\right\|_{i \infty} & \leq \zeta_{V 1}\|a\| \\
\left\|V_{m}(q, a)-V_{m}(q, b)\right\|_{i \infty} & \leq \zeta_{V 2}\|a-b\| \\
\left\|F_{d}\right\|_{i \infty} & \leq \zeta_{F 1} \\
\|G(a)-G(b)\| & \leq \zeta_{G 1}\|a-b\|
\end{aligned}
$$

$\forall a, b \in \mathbb{R}^{n}$ where $\zeta_{V_{1}}, \zeta_{V_{2}}, \zeta_{G 1}, \zeta_{F 1}$ are known positive bounding constants.

\section{Control ObJective And Design}

The control objective can be stated as ensuring the endeffector of robot manipulator to track a desired task space trajectory, i.e., make $x(t) \rightarrow x_{d}(t)$ where $x_{d}(t) \in \mathbb{R}^{n}$ is the desired trajectory which is assumed to be chosen as sufficiently smooth with bounded time derivatives. The control problem is further restricted by the unavailability of joint velocity measurements. In order to quantify the control objective we define the task-space tracking error signal $e(t) \in \mathbb{R}^{n}$ as

$$
e \triangleq x_{d}-x .
$$

To remove the joint velocity measurements dependencies in the controller design, we have formulated the following novel dynamic model free joint velocity observer design as

$$
\begin{aligned}
\dot{\hat{q}}= & y+K_{o 1} \tilde{q}+K_{c} q \\
\dot{y}= & \left(K_{o 2}+I_{n}\right) \tilde{q}+K_{o 3} \operatorname{Sgn}(\tilde{q}) \\
& -K_{c} J^{-1}\left(\dot{x}_{d}+\alpha e\right)
\end{aligned}
$$


where $K_{o 1}, K_{o 2}, K_{o 3}, K_{c} \in \mathbb{R}^{n \times n}$ are constant, positive definite, diagonal observer and controller gain matrices, $\tilde{q} \in$ $\mathbb{R}^{n}$ is the joint position observation error explicitly defined as

$$
\tilde{q} \triangleq q-\hat{q}
$$

with $\hat{q}(t) \in \mathbb{R}^{n}$ being the observed joint position and $\operatorname{Sgn}(\cdot) \in \mathbb{R}^{n}$ is the vector signum function and $y(t) \in \mathbb{R}^{n}$ is an auxiliary term.

\section{A. Error Dynamics}

We start our controller development by taking the time derivative of (23) and substituting (2) for $\dot{x}$ to obtain

$$
\dot{e}=\dot{x}_{d}-\alpha e+\alpha e-J \dot{q}
$$

where $\alpha \in \mathbb{R}$ is a positive constant gain. Based on the subsequent analysis we define an auxiliary term, $r(t) \in \mathbb{R}^{n}$, as

$$
r \triangleq J^{-1}\left(\dot{x}_{d}+\alpha e\right)-\dot{q}
$$

which enables us to rewrite the error dynamics in the form

$$
\dot{e}=-\alpha e+J r
$$

We would like to note that as the auxiliary term $r(t)$ in (28) has $\dot{q}(t)$ term, therefore is not available for control design. However based on the structure of $\dot{e}$ given in (29), in order to regulate $e(t)$, we need to regulate $r(t)$, therefore we also need to calculate the dynamics for $r(t)$. To this end we take the time derivative of (28), pre-multiply the resultant by $M(q)$ and apply (12) to obtain

$$
\begin{aligned}
M \dot{r} & =M(q) \frac{d}{d t}\left\{J^{-1}(q)\left(\dot{x}_{d}+\alpha e\right)\right\} \\
& +V_{m}(q, \dot{q}) \dot{q}+G(q)+F_{d} \dot{q}-\tau .
\end{aligned}
$$

Adding/subtracting $V_{m}(q, \dot{q}) r$ to (30) and re-arranging we obtain

$$
M \dot{r}=-V_{m}(q, \dot{q}) r-\tau+\Omega_{1}+\Omega_{2}
$$

where the auxiliary terms $\Omega_{1}\left(q, \hat{q}, \dot{\hat{q}}, x_{d}, \dot{x}_{d}, \ddot{x}_{d}\right) \in \mathbb{R}^{n}$ and $\Omega_{2}\left(q, \dot{\tilde{q}}, x_{d}, \dot{x}_{d}\right) \in \mathbb{R}^{n}$ are explicitly defined as

$$
\begin{aligned}
\Omega_{1} \triangleq & M(q) W_{j}\left(q,\left(\dot{x}_{d}+\alpha e\right)\right) \dot{\hat{q}} \\
& +M(q) J^{-1}(q)\left(\ddot{x}_{d}+\alpha \dot{x}_{d}-\alpha J(q) \dot{\hat{q}}\right) \\
& +V_{m}(q, \dot{\hat{q}}) J^{-1}(q)\left(\dot{x}_{d}+\alpha e\right) \\
& +F_{d} \dot{\hat{q}}+G(q),
\end{aligned}
$$

$$
\begin{aligned}
\Omega_{2} \triangleq & M(q) W_{j}\left(q,\left(\dot{x}_{d}+\alpha e\right)\right) \dot{\tilde{q}}-\alpha M(q) \dot{\tilde{q}} \\
& +V_{m}(q, \dot{\tilde{q}}) J^{-1}(q)\left(\dot{x}_{d}+\alpha e\right)+F_{d} \dot{\tilde{q}}
\end{aligned}
$$

We would like to note that $\Omega_{1}$ term contains known and measurable terms while $\Omega_{2}$ contains the unmeasurable terms. Furthermore by exploiting the properties of the robot dynamics and assumptions we made on the kinematics we can show that,

$$
\left\|\Omega_{2}\right\| \leq \rho_{c}(\|z\|)\|z\|
$$

where $z(t) \in \mathbb{R}^{4 n}$ is defined as

$$
z(t)=\left[e^{T}(t) r^{T}(t) \tilde{q}^{T}(t) s^{T}(t)\right]^{T}
$$

with $\rho_{c}(\cdot) \in \mathbb{R}^{1}$ being a known, positive bounding function and $s(t) \in \mathbb{R}^{n}$ is the auxiliary observation error term defined as

$$
s \triangleq \dot{\tilde{q}}+\alpha \tilde{q}
$$

Given the open-loop error system given in (31) and from the subsequent stability analysis we design the control input torque signal as

$$
\tau=\Omega_{1}+K_{c}\left(J^{-1}(q)\left(\dot{x}_{d}+\alpha e\right)-\dot{\hat{q}}+\alpha \tilde{q}\right)+J^{T} e
$$

where $K_{c} \in \mathbb{R}^{n \times n}$ is a constant, positive definite, diagonal control gain matrix. Using the fact

$$
J^{-1}\left(\dot{x}_{d}+\alpha e\right)-\dot{\hat{q}}+\alpha \tilde{q}=r+s
$$

we can rewrite the control input signal, as

$$
\tau=\Omega_{1}+K_{c}(r+s)+J^{T} e .
$$

For the ease of presentation, we will use the unimplementable definition (39) in our analysis however on actual implementations (37) is used. To develop the closed-loop error system we substitute (39) into (31) to obtain

$$
M \dot{r}=-V_{m}(q, \dot{q}) r+\Omega_{2}-K_{c}(r+s)-J^{T} e .
$$

\section{B. Observer Analysis}

We start the observation error system analysis by taking the time derivative of (36), and insert (24), (26) to obtain

$$
\begin{aligned}
\dot{s}= & M^{-1}\left[\tau-V_{m} \dot{q}-G-F_{d} \dot{q}\right]+K_{c} r \\
& -\left(K_{o 1}-\alpha\right) \dot{\tilde{q}}-K_{o 2} \tilde{q}-\tilde{q}-K_{o 3} \operatorname{Sgn}(\tilde{q})
\end{aligned}
$$

where (12) and (28) were used. Selecting the observer gains to satisfy $K_{o 2}=\alpha\left(K_{o 1}-\alpha I_{n}\right)$ and inserting for the control input torque of (39), we can rewrite the dynamics of the auxiliary filtered observation error term as

$$
\begin{aligned}
\dot{s} & =N_{d}+N_{b} \\
& +K_{c} r-\frac{K_{o 2}}{\alpha} s-\tilde{q}-K_{o 3} \operatorname{Sgn}(\tilde{q})
\end{aligned}
$$

where we have auxiliary term $N_{d}\left(x_{d}, \dot{x}_{d}, t\right) \in \mathbb{R}^{n}$, contains all the terms that are desired end-effector position and velocity dependent. Due to the boundedness of the desired trajectory and its time derivatives the $N_{d}$ term and its time derivative can also be bounded as

$$
\left\|N_{d i}\right\| \leq \zeta_{N 1 i},\left\|\dot{N}_{d i}\right\| \leq \zeta_{N 2 i}
$$

where $\zeta_{N 1 i}, \zeta_{N 2 i} \forall i=1,2, \cdots, n$ are positive bounding constants. The other auxiliary term, $N_{b}(t) \in \mathbb{R}^{n}$, also introduced in (42) is used to represent the rest of the terms which can be bounded in the form

$$
\left\|N_{b}\right\| \leq \rho_{o}(\|z\|)\|z\|
$$

where $\rho_{o}(\cdot) \in \mathbb{R}^{1}$ is a known positive bounding function and $z(t)$ was previously defined. 
Remark 1: While the bounds in (44) are assumed to be known, in reality usually upper bounds of these bounds are sufficient for gain tuning.

Obtaining the closed-loop observation error dynamics of (42) enables us to state a preliminary stability analysis for the observation system. We start our preliminary analysis by defining a non-negative scalar function $V_{o}(t)$ as follows,

$$
V_{o} \triangleq \frac{1}{2} s^{T} s+\frac{1}{2} \tilde{q}^{T} \tilde{q}+P
$$

where the scalar auxiliary function $P(t) \in \mathbb{R}$ is defined as

$$
P \triangleq \zeta_{P}-\int_{0}^{t} s^{T}(\sigma)\left[N_{d}(\sigma)-K_{o 3} \operatorname{Sgn}(\tilde{q}(\sigma))\right] d \sigma
$$

with the non-negative constant $\zeta_{P}$ defined as

$$
\zeta_{P} \triangleq \sum_{i=1}^{n} K_{o 3 i}\left|\tilde{q}_{i}(0)\right|-\tilde{q}^{T}(0) N_{d}(0)
$$

where the subscript $i=1,2, \ldots, n$ denotes the $i^{\text {th }}$ element of a vector. Following a similar analysis to that of [28], it can be proven that when the observer gain $K_{o 3}$ is selected to satisfy the following sufficient condition

$$
K_{o 3 i} \geq \zeta_{N 1 i}+\frac{1}{\alpha} \zeta_{N 2 i} \forall i=1, \cdots, n
$$

then $P(t)$ defined in (46) is always greater or equal to zero and therefore $V_{o}$ defined in (45) is a positive definite Lyapunov function with respect to $s, \tilde{q}$ and $\sqrt{P}$. Taking the time derivative of (45) and substituting for (36), (42) and time derivative of (46) we obtain

$$
\dot{V}_{o}=-s^{T}\left[\frac{K_{o 2}}{\alpha} s+N_{b}+K_{c} r\right]-\alpha\|\tilde{q}\|^{2} .
$$

The first term in the brackets and the last term of equation (49) are designed to ensure the stability and convergence of the filtered version and the actual the observation error signals, while the rest of the terms are designed to cancel the interconnection terms between the observer/controller subsystem. We are now ready to pursue to the overall stability analysis of the observer/controller couple.

\section{Stability AnALYSiS}

The error dynamics of (29), the closed-loop dynamics for $r(t)$ obtained at (40) and the result of the preliminary observer stability analysis presented in the previous subsection yield the following result for the position tracking and observation error terms.

Theorem 1: The controller formulation of (37), and the velocity observer formulation given in (24), (26) ensure that the closed loop observer/controller is asymptotically stable. That is

$$
\|e(t)\|,\|\tilde{q}(t)\| \rightarrow 0 \text { as } t \rightarrow \infty,
$$

provided that following gain conditions with (48) are satisfied

$$
\begin{aligned}
\lambda_{\min }\left\{K_{o 2}\right\} & \geq \alpha\left(\alpha+k_{n} \rho_{o}^{2}\right), \\
\lambda_{\min }\left\{K_{c}\right\} & \geq\left(\alpha+k_{n} \rho_{c}^{2}\right)
\end{aligned}
$$

with the control gain $\alpha$ selected to satisfy $\alpha \gg \frac{1}{2 k_{n}}$.
Proof: We start our analysis by defining non-negative function $V(t) \in \mathbb{R}$ as follows

$$
V \triangleq V_{o}+\frac{1}{2} r^{T} M(q) r+\frac{1}{2} e^{T} e
$$

where $V_{o}(t)$ was previously defined in (45). Note that (53) can be upper and lower bounded as

$$
\lambda_{1}\|z\|^{2} \leq \lambda_{1}\|y\|^{2} \leq V \leq \lambda_{2}\|y\|^{2}
$$

where, $\lambda_{1}, \lambda_{2}$ are some bounding constants, $z(t)$ was defined in (35), and $y(t) \in \mathbb{R}^{4 n+1}$ is defined as

$$
y \triangleq\left[\begin{array}{lllll}
e^{T} & r^{T} & \tilde{q}^{T} & s^{T} & \sqrt{P}
\end{array}\right]^{T} .
$$

Taking the time derivative of (53), substituting for (29), (40), (49) and canceling common terms, we obtain

$$
\begin{aligned}
\dot{V}= & -s^{T}\left[\frac{K_{o 2}}{\alpha} s+N_{b}\right]-\alpha\|\tilde{q}\|^{2} \\
& -r^{T}\left[\Omega_{2}-K_{c} r\right]-\alpha\|e\|^{2}
\end{aligned}
$$

where Property 4 was made use of as well. Applying (44) and (34) we can obtain an upper bound for (56) as follows

$$
\begin{aligned}
\dot{V} \leq & -\frac{1}{\alpha} \lambda_{\min }\left\{K_{o 2}\right\}\|s\|^{2}+\rho_{o}\|z\|\|s\|-\alpha\|\tilde{q}\|^{2} \\
& -\lambda_{\min }\left\{K_{c}\right\}\|r\|^{2}+\rho_{c}\|z\|\|r\|-\alpha\|e\|^{2}
\end{aligned}
$$

which using the definition of $z(t)$ given in (35), the gain condition of (51) and (52) along with the damping argument

$$
\|a\|\|b\|-k_{n}\|a\|^{2} \leq \frac{1}{4 k_{n}}\|b\|^{2} \quad \forall a, b \in \mathbb{R}^{n}
$$

we can further upper bound the time derivative of $V(t)$ to have the form

$$
\dot{V} \leq-\left(\alpha-\frac{1}{2 k_{n}}\right)\|z\|^{2} .
$$

From (53) and (59) it is clear that when the gain condition on $k_{n}$ and $\alpha$ is satisfied, $e(t), r(t), s(t), \tilde{q}(t) \in \mathcal{L}_{\infty} \cap$ $\mathcal{L}_{2}$, and following standard signal chasing arguments we can prove that all signals in the closed loop error and observation system are bounded. Based on the fact that $r(t), s(t) \in \mathcal{L}_{\infty}$ we can conclude that $e(t)$ and $\dot{\tilde{q}}(t)$ are uniformly continuous. Finally from direct application of Barbalat's Lemma [29] $\|z(t)\| \rightarrow 0$ as time increases so we can obtain the result given in (50) provided that the gain conditions given in the theorem are satisfied.

Remark 2: Due to the structure of the bounding functions $\rho_{c}(\cdot)$ and $\rho_{o}(\cdot)$ given in (34) and (44) respectively, and from the presentation of the Theorem, one might come to the conclusion that the controller/observer formulation requires the norms of $r(t)$ and $s(t)$ (as $z(t)$ defined in (35) contains these signals). We would like to note that the controller/observer formulation does not make use the bounding functions as a whole but uses the coefficients of these functions. The proof presented here is constructed to ease the presentation of the work by removing the tedious parts. Following a similar approach to [30] and [31], obtaining the same stability result is trivial. 


\section{Simulation Results}

We have constructed our numerical studies on the dynamic model of a two degree of freedom planar robot manipulator with the following parameters

$$
\begin{aligned}
M & =\left[\begin{array}{cc}
p_{2}+p_{3}+2 p_{1} s_{2} & p_{2}+p_{1} s_{2} \\
p_{2}+p_{1} s_{2} & p_{2}
\end{array}\right] \\
V_{m} & =\left[\begin{array}{cc}
p_{1} s_{2} \dot{q}_{2} & p_{1} s_{2}\left(\dot{q}_{1}+\dot{q}_{2}\right) \\
-p_{1} s_{2} \dot{q}_{1} & 0
\end{array}\right] \\
G & =\left[\begin{array}{c}
p_{4} c_{1}+p_{5} c_{12} \\
p_{5}
\end{array}\right] \\
F_{d} & =\left[\begin{array}{cc}
p_{6} & 0 \\
0 & p_{7}
\end{array}\right]
\end{aligned}
$$

in which $s_{2}=\sin \left(q_{2}\right), c_{2}=\cos \left(q_{2}\right), c_{12}=\cos \left(q_{1}+q_{2}\right)$, $p_{1}=0.36, p_{2}=0.43, p_{3}=0.93, p_{4}=22.344, p_{5}=5.88$, $p_{6}=1, p_{7}=1$. For the simulations the desired task space, end-effector velocity, $\dot{x}_{d}$, was selected as

$$
\dot{x}_{d}=\left[\begin{array}{c}
-0.05 \sin (0.1 t) \\
0.04 \cos (0.2 t)
\end{array}\right]
$$

where the robot manipulator was considered to be at rest with the initial joint position as $q(0)=[0.4,0.4]^{T}$ rad. After a rough tuning process, satisfactory tracking performance was obtained when the control gains were selected $\alpha=1, K_{o 1}=$ $40, K_{o 3}=5$ and $K_{c}=10$. The corresponding results are presented in Figures 1-4.

In Figure 1, the task space position tracking error is presented while $x_{d}(t)$ vs $x(t)$ is given in Figure 2. The joint position observer error is given in Figure 3 . And finally Figure 4 presents the control input torques.
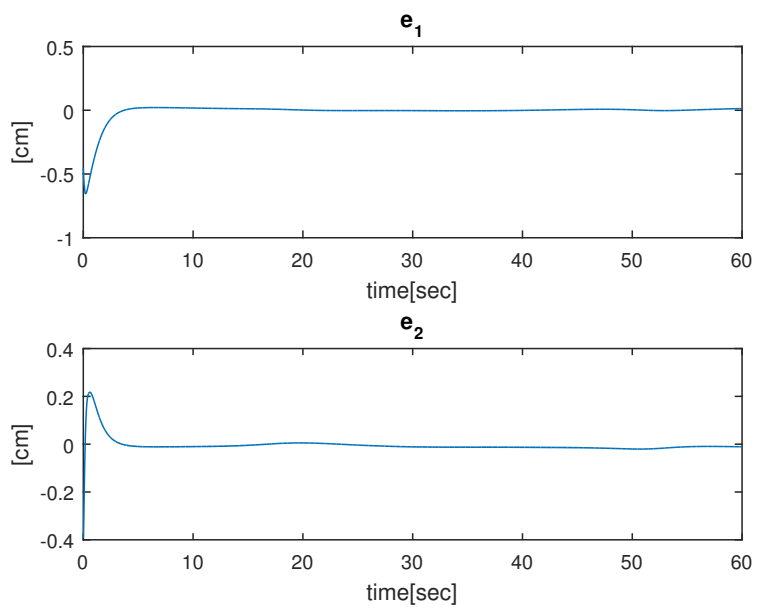

Fig. 1. Task space position tracking error $e(t)$

From these preliminary results we can conclude that the controller/observation objectives are met.

\section{CONCLUSIONS}

In this work, we presented the design and the corresponding stability analysis of a dynamical model independent observer based output feedback end-effector tracking

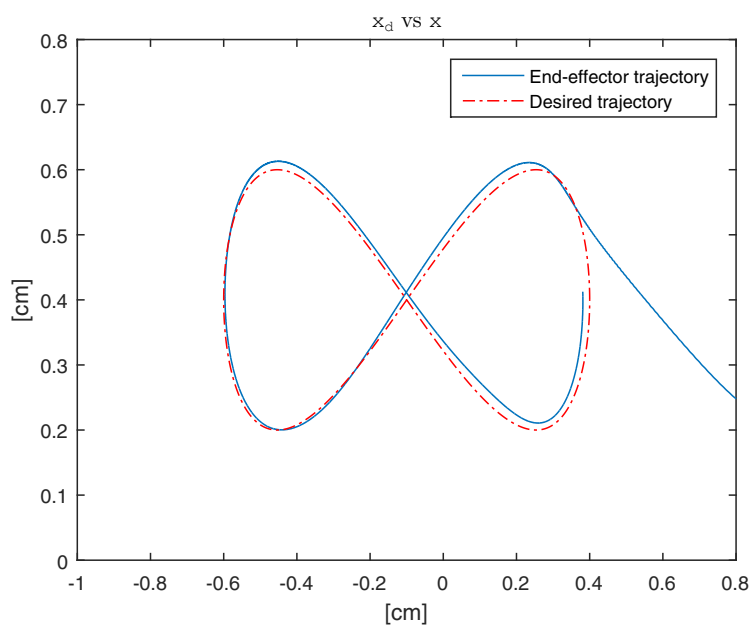

Fig. 2. $x_{d}(t)$ vs $x(t)$
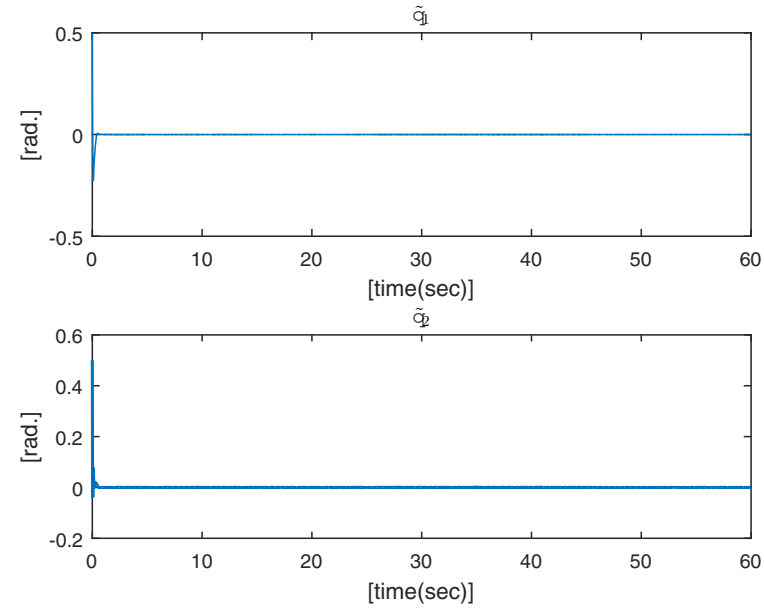

Fig. 3. Joint position observation error $\tilde{q}(t)$

controller for robotic manipulators. The proposed method, due to the structure of the observer formulation can be extended for controller formulation where the system model is uncertain or simply unavailable. Simulation results are provided to illustrate the performance of the proposed controller/observer couple. Future work will concentrate on adaptive and learning controller based extensions with the same velocity observer design.

The proposed strategy is compared with some of the works in the literature. In [28], a full-state feedback controller for MIMO systems was designed where only signum function usage in this paper was inspired from. In [30] and [31], the designed controllers were for joint space control of robot manipulators where task space control was aimed in this paper.

\section{REFERENCES}

[1] L. Sciavicco and B. Siciliano, Modeling and Control of Robot Manipulators. New York, NY: McGraw-Hill Co., 1996.

[2] J. Peters and S. Schaal, "Learning to control in operational space," Int. J. Robotics Research, vol. 27, no. 2, pp. 197-212, 2008 

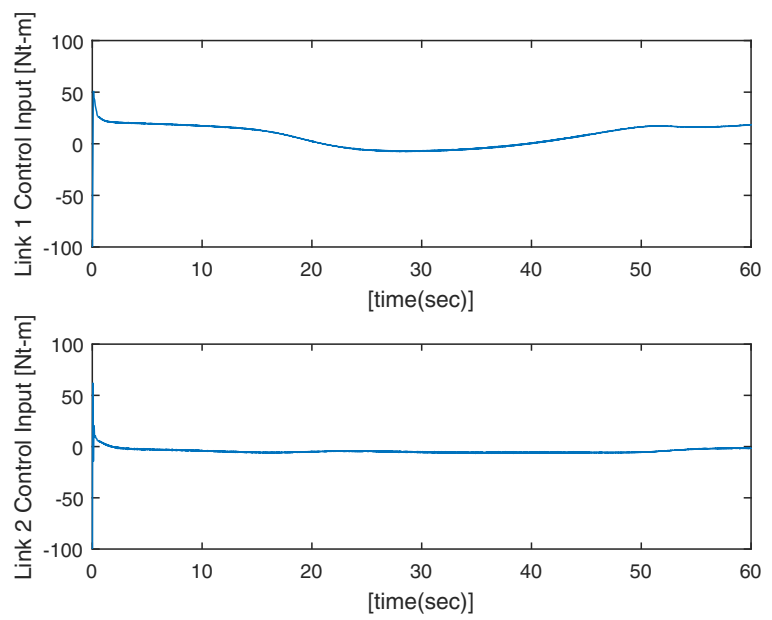

Fig. 4. Control input torque $\tau(t)$

[3] J. Nakanishi, R. Cory, M. Mistry, J. Peters, and S. Schaal, "Operational space control: A theoretical and empirical comparison," Int. J. Robotics Research, vol. 27, no. 6, pp. 737-757, 2008.

[4] O. Khatib, "Dynamic control of manipulators in operational space," in IFTOMM Cong. Theory of Machines and Mechanisms, New Delhi, India, 1983, pp. 1-10.

[5] P. Hsu, J. Hauser, and S. Sastry, "Dynamic control of redundant manipulators," J. of Robotic Systems, vol. 6, no. 3, pp. 133-148, 1989.

[6] E. Tatlicioglu, M. L. McIntyre, D. M. Dawson, and I. D. Walker, "Adaptive non-linear tracking control of kinematically redundant robot manipulators," Int. J. of Robotics and Automation, vol. 23, no. 2, pp. 98-105, 2008.

[7] E. Tatlicioglu, D. Braganza, T. C. Burg, and D. M. Dawson, "Adaptive control of redundant robot manipulators with sub-task extensions," Robotica, vol. 27, no. 6, pp. 873-881, 2009.

[8] H. Yazarel and C. C. Cheah, "Task-space adaptive control of robotic manipulators with uncertainties in gravity regressor matrix and kinematics," IEEE Tr. on Automatic Control, vol. 47, pp. 1580-1585, September 2002.

[9] C. C. Cheah, S. Kawamura, S. Arimoto, and K. Lee, "PID control of robotic manipulator with uncertain jacobian matrix," in Proc. IEEE Int. Conf. Robot. Autom., Detroit, MI, USA, 1999, pp. 494-499.

[10] C. C. Cheah, K. Lee, S. Kawamura, and S. Arimoto, "Asymptotic stability of robot control with approximate jacobian matrix and its application to visual servoing," in Proc. IEEE Int. Conf. Decision and Control, Sydney, Australia, 2000, pp. 3939-3944.

[11] C. C. Cheah, S. Kawamura, S. Arimoto, and K. Lee, "Tuning for taskspace feedback control of robot with uncertain jacobian matrix," IEEE Tr. on Automatic Control, vol. 46, pp. 1313-1318, August 2001.

[12] C. C. Cheah, "Approximate jacobian robot control with adaptive jacobian matrix," in Proc. IEEE Int. Conf. Decision and Control, Maui, HI, USA, 2003, pp. 5859-5864.

[13] C. C. Cheah, M. Hirano, S. Kawamura, and S. Arimoto, "Approximate jacobian control with task-space damping for robot manipulators," IEEE Tr. on Automatic Control, vol. 49, no. 5, pp. 752-757, 2004.

[14] C. C. Cheah, C. Liu, and J. J. E. Slotine, "Adaptive tracking control for robots with unknown kinematic and dynamic properties," Int. J. Robotics Research, vol. 25, no. 3, pp. 283-296, 2006.

[15] D. D. Braganza, W. E. Dixon, D. M. Dawson, and B. Xian, "Tracking control for robot manipulators with kinematic and dynamic uncertainty," Int. J. of Robotics and Automation, vol. 23, no. 2, pp. 117-126, 2008.

[16] K. M. Dogan, E. Tatlicioglu, and E. Zergeroglu, "Operational/task space learning control of robot manipulators with dynamical uncertainties," in Proc. of IEEE Int. Conf. on Control Applications, Sydney, Australia, 2015, pp. 527-532.

[17] U. Ozbay, H. T. Sahin, and E. Zergeroglu, "Robust tracking control of kinematically redundant robot manipulators subject to multiple selfmotion criteria," Robotica, vol. 26, no. 6, pp. 711-728, 2008.

[18] K. Cetin, E. Tatlicioglu, and E. Zergeroglu, "Continuous robust task- space tracking control of robotic manipulators with uncertain dynamics," in Proc. of IEEE Int. Conf. on Control Applications, Sydney, Australia, 2015, pp. 312-317.

[19] M. Galicki, "Constraint finite-time control of redundant manipulators," Int. J. of Robust \& Nonlinear Control, 2016.

[20] _ - "Finite-time trajectory tracking control in a task space of robotic manipulators," Automatica, vol. 67, pp. 165-170, 2016.

[21] _ "Robust task space finite-time chattering-free control of robotic manipulators," J. Intelligent and Robotic Systems, 2016.

[22] E. Zergeroglu, D. Dawson, I. D. Walker, and A. Behal, "Nonlinear tracking control of kinematically redundant robot manipulators," in Proc. American Control Conf., Chicago, IL, USA, 2000, pp. 25132517.

[23] E. Zergeroglu, D. M. Dawson, I. D. Walker, and P. Setlur, "Nonlinear tracking control of kinematically redundant robot manipulators," IEEE/ASME Tr. on Mechatronics, vol. 9, no. 1, pp. 129-132, 2004.

[24] B. Xian, M. S. de Queiroz, D. M. Dawson, and I. D. Walker, "Taskspace tracking control of robot manipulators via quaternion feedback," IEEE Tr. on Robotics and Automation, vol. 20, no. 1, pp. 160-167, 2004.

[25] H. K. Khalil, Nonlinear Systems, 3rd Edition. New York, NY, USA: Prentice Hall, 2002.

[26] D. M. Dawson, M. M. Bridges, and Z. Qu, Nonlinear Control of Robotic Systems for Environmental Waste and Restoration. Englewood Cliffs, NJ, USA: Prentice-Hall, 1995.

[27] F. L. Lewis, D. M. Dawson, and C. T. Abdallah, Robot Manipulator Control: Theory and Practice. New York, NY, USA: Marcel Dekker, Inc., 2004.

[28] B. Xian, M. S. de Queiroz, and D. M. Dawson, "A continuous control mechanism for uncertain nonlinear systems," in Control, Stabilization, and Nonsmooth Analysis, Lecture Notes in Control and Information Sciences. Heidelberg, Germany: Springer-Verlag, 2004, pp. 251-262.

[29] M. Krstic, I. Kanellakopoulos, and P. Kokotovic, Nonlinear and Adaptive Control Design. New York, NY, USA: John Wiley \& Sons, 1995.

[30] E. Zergeroglu and E. Tatlicioglu, "Observer based output feedback tracking control of robot manipulators," in Proc. of IEEE Int. Conf. on Control Applications, Yokohoma, Japan, 2010, pp. 602-607.

[31] E. Zergeroglu, E. Tatlicioglu, and E. Kaleli, "A model independent observer based output feedback tracking controller for robotic manipulators with dynamical uncertainties," Robotica, vol. 35, no. 4, pp. 729-743, 2017. 